\title{
ON EQUIVALENCES FOR COHOMOLOGICAL MACKEY FUNCTORS
}

\author{
MARKUS LINCKELMANN
}

\begin{abstract}
By results of Rognerud, a source algebra equivalence between two $p$-blocks of finite groups induces an equivalence between the categories of cohomological Mackey functors associated with these blocks, and a splendid derived equivalence between two blocks induces a derived equivalence between the corresponding categories of cohomological Mackey functors. We prove this by giving an intrinsic description of cohomological Mackey functors of a block in terms of the source algebras of the block, and then using this description to construct explicit two-sided tilting complexes realising the above mentioned derived equivalence. We show further that a splendid stable equivalence of Morita type between two blocks induces an equivalence between the categories of cohomological Mackey functors which vanish at the trivial group. We observe that the module categories of a block, the category of cohomological Mackey functors, and the category of cohomological Mackey functors which vanish at the trivial group arise in an idempotent recollement. Finally, we extend a result of Tambara on the finitistic dimension of cohomological Mackey functors to blocks.
\end{abstract}

\section{INTRODUCTION}

Let $p$ be a prime and $\mathcal{O}$ a complete local principal ideal domain with residue field $k=\mathcal{O} / J(\mathcal{O})$ of characteristic $p$; we allow the case $\mathcal{O}=k$. Let $G$ be a finite group. The blocks of $\mathcal{O} G$ are the primitive idempotents in $Z(\mathcal{O} G)$. By a result of Yoshida in [13, the category $\operatorname{coMack}(\mathcal{O} G)$ of cohomological Mackey functors of $G$ with coefficients in $\bmod (\mathcal{O})$ is equivalent to the category of finitely generated modules over the algebra $\operatorname{End}_{\mathcal{O} G}\left(\oplus_{H} \mathcal{O} G / H\right)^{\text {op }}$, where $H$ runs over the subgroups of $G$. This description implies that the category $\operatorname{coMack}(\mathcal{O} G)$ decomposes as a direct sum of the categories $\operatorname{coMack}(G, b)$, with $b$ running over the blocks of $\mathcal{O} G$, such that each $\operatorname{coMack}(G, b)$ is equivalent to the category of finitely generated modules over the algebra $\operatorname{End}_{\mathcal{O} G}\left(\oplus_{H} b \cdot \mathcal{O} G / H\right)^{\mathrm{op}}$, where $H$ runs as before over the subgroups of $G$. By results of Thévenaz and Webb in [12, §17], this is the block decomposition of $\operatorname{coMack}(\mathcal{O} G)$. We use Yoshida's result to describe $\operatorname{coMack}(G, b)$ in an analogous way at the source algebra level, obtaining as a consequence an alternative proof of a result of Rognerud in [10, stating that blocks with isomorphic source algebras have equivalent categories of cohomological Mackey functors. A defect group of a block $b$ of $\mathcal{O} G$ is a maximal $p$-subgroup $P$ of $G$ such that $\mathcal{O} P$ is isomorphic to a direct summand of $\mathcal{O} G b$ as an $\mathcal{O} P-\mathcal{O} P$-bimodule. A source idempotent of $b$ is a primitive idempotent $i$ in the fixed point algebra $(\mathcal{O} G b)^{P}$ with respect to the conjugation action of $P$ on $\mathcal{O} G b$ such that $\mathcal{O} P$ is isomorphic to a direct summand of $i \mathcal{O} G i$ as

Received by the editors October 6, 2015 and, in revised form, April 13, 2016.

2010 Mathematics Subject Classification. Primary 20 J05. 
an $\mathcal{O} P$ - $\mathcal{O} P$-bimodule. The algebra $A=i \mathcal{O} G i$ with its canonical image $P i$ of $P$ in $A^{\times}$is called a source algebra of $b$.

Theorem 1.1. Let $G$ be a finite group, $b$ a block of $\mathcal{O} G$ with a defect group $P$ and let $A$ be a source algebra of $b$. Set $N=\bigoplus_{Q} A \otimes_{\mathcal{O} Q} \mathcal{O}$, where in the direct sum $Q$ runs over the subgroups of $P$. Set $E=\operatorname{End}_{A}(N)$. There is an equivalence of categories $\operatorname{coMack}(G, b) \cong \bmod \left(E^{\mathrm{op}}\right)$. In particular, blocks with isomorphic source algebras have equivalent categories of cohomological Mackey functors.

By a result of Rognerud [10, Proposition 4.5], the last statement holds more generally for permeable Morita equivalences; that is, Morita equivalences preserving $p$-permutation modules (and the proof presented below shows this as well). It is not necessary to take the direct sum over all subgroups of $P$ in the statement of 1.1 . Indeed, by [5, Proposition 6.1], every indecomposable direct summand of $A \otimes_{\mathcal{O} Q} \mathcal{O}$ for some subgroup $Q$ of $P$ is isomorphic to a direct summand of $A \otimes_{\mathcal{O} R} \mathcal{O}$ for some fully centralised subgroup $R$ with respect to the fusion system $\mathcal{F}$ determined by $A$ on $P$. Thus we obtain a Morita equivalent endomorphism algebra if we take the direct sum over a set of representatives of the $\mathcal{F}$-isomorphism classes of fully $\mathcal{F}$-centralised subgroups of $P$.

At the source algebra level, a splendid derived equivalence between two source algebras $A, A^{\prime}$ of blocks with a common defect group $P$ is a derived equivalence induced by a splendid Rickard complex of $A$ - $A^{\prime}$-bimodules $X$; that is, $X$ is a bounded chain complex of $A$ - $A^{\prime}$-bimodules whose terms are direct sums of summands of the bimodules $A \otimes_{\mathcal{O} Q} A^{\prime}$, where $Q$ runs over the subgroups of $P$, such that we have homotopy equivalences $X \otimes_{A^{\prime}} X^{*} \simeq A$ and $X^{*} \otimes_{A} X \simeq A^{\prime}$ as complexes of $A$ $A$-bimodules and of $A^{\prime}$ - $A^{\prime}$-bimodules, respectively. Here $X^{*}$ denotes the $\mathcal{O}$-dual of $X$, rewritten as a chain complex. Rognerud showed in 10 that a splendid Rickard equivalence between two blocks induces a derived equivalence between the associated categories of cohomological Mackey functors. We construct two-sided complexes which realise this derived equivalence.

Theorem 1.2. Let $A, A^{\prime}$ be source algebras of blocks $b, b^{\prime}$ of finite group algebras $\mathcal{O} G, \mathcal{O} G^{\prime}$, respectively, with a common defect group $P$. Set $N=\bigoplus_{Q} A \otimes_{\mathcal{O} Q} \mathcal{O}$ and $N^{\prime}=\bigoplus_{Q} A^{\prime} \otimes_{\mathcal{O} Q} \mathcal{O}$, where $Q$ runs over the subgroups of $P . \operatorname{Set} E=\operatorname{End}_{A}(N)$ and $E^{\prime}=\operatorname{End}_{A^{\prime}}\left(N^{\prime}\right)$. Let $X$ be a splendid Rickard complex of $A$ - $A^{\prime}$-bimodules. Set $Y=\operatorname{Hom}_{A}\left(X \otimes_{A^{\prime}} N^{\prime}, N\right)$ and $Y^{\prime}=\operatorname{Hom}_{A^{\prime}}\left(X^{*} \otimes_{A} N, N^{\prime}\right)$. Then $Y$ is a complex of E-E'-bimodules, $Y^{\prime}$ is a complex of $E^{\prime}$-E-bimodules, the terms of $Y$ and $Y^{\prime}$ are finitely generated projective as left and as right modules, and we have homotopy equivalences

$$
\begin{aligned}
& Y \otimes_{E^{\prime}} Y^{\prime} \simeq E, \\
& Y^{\prime} \otimes_{E} Y \simeq E^{\prime}
\end{aligned}
$$

as complexes of E-E-bimodules and $E^{\prime}$-E'-bimodules, respectively. In particular, if $\mathcal{O} G b$ and $\mathcal{O} G^{\prime} b^{\prime}$ are splendidly Rickard equivalent, then the categories $\operatorname{coMack}(G, b)$ and $\operatorname{coMack}\left(G^{\prime}, b^{\prime}\right)$ are derived equivalent.

As mentioned above, the last statement of 1.2 is due to Rognerud [10, Theorem 5.8], where, as before, this is shown to hold more generally for permeable derived equivalences (that is, derived equivalences induced by Rickard complexes which preserve complexes of $p$-permutation modules). The first statement of 1.2 is a special 
case of a more general construction of derived equivalences for endomorphism algebras, which we will describe in the next section (and which also allows for the extra generality regarding permeable derived equivalences).

There are versions of the above results for stable endomorphism algebras and stable equivalences. Let $A$ be an $\mathcal{O}$-algebra. An $A$-module $U$ is relatively $\mathcal{O}$ projective if $U$ is isomorphic to a direct summand of $A \otimes_{\mathcal{O}} W$ for some $\mathcal{O}$-module $W$. For any two $A$-modules $U, U^{\prime}$ we denote by $\operatorname{Hom}_{A}^{\mathrm{pr}}\left(U, U^{\prime}\right)$ the subspace of $\operatorname{Hom}_{A}\left(U, U^{\prime}\right)$ consisting of all $A$-homomorphisms which factor through a relatively $\mathcal{O}$-projective $A$-module. We set $\underline{\operatorname{Hom}}_{A}\left(U, U^{\prime}\right)=\operatorname{Hom}_{A}\left(U, U^{\prime}\right) / \operatorname{Hom}_{A}^{\mathrm{pr}}\left(U, U^{\prime}\right)$. We denote by $\operatorname{stmod}(A)$ the $\mathcal{O}$-linear category having the finitely generated $A$-modules as objects, with homomorphism spaces $\underline{\operatorname{Hom}}_{A}\left(U, U^{\prime}\right)$ for any two finitely generated $A$-modules $U, U^{\prime}$, such that the composition of $\operatorname{morphisms}$ in $\operatorname{stmod}(A)$ is induced by the composition of $A$-homomorphisms (one checks that this is well-defined). The resulting category is no longer abelian, but if $A$ is symmetric, then this category can be shown to be triangulated (a fact we will not need in this paper). For $G$ a finite group and $b$ a block of $\mathcal{O} G$, we denote by $\operatorname{coMack}_{0}(G, b)$ the full subcategory of $\operatorname{coMack}(G, b)$ consisting of all Mackey functors in $\operatorname{coMack}(G, b)$ whose evaluation at the trivial subgroup $\left\{1_{G}\right\}$ of $G$ is zero.

Theorem 1.3. Let $G$ be a finite group, $b$ a block of $\mathcal{O} G$ with a defect group $P$ and let $A$ be a source algebra of $b$. Set $N=\bigoplus_{Q} A \otimes_{\mathcal{O} Q} \mathcal{O}$, where $Q$ runs over the subgroups of $P$. Set $\underline{E}=\underline{\operatorname{End}}_{A}(N)$. There is an equivalence of categories $\left.\operatorname{coMack}_{0}(G, b) \cong \bmod \left(\underline{E}^{\mathrm{op}}\right)\right)$.

Corollary 1.4. With the notation above, there is a recollement of abelian categories

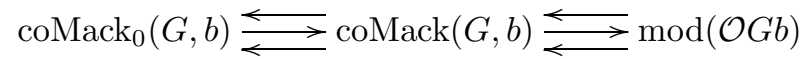

which is isomorphic to the idempotent recollement of the algebra $E$ with the idempotent $\tau$, where $\tau$ is the canonical projection of $N$ onto its summand $A=A \otimes_{\mathcal{O}} \mathcal{O}$.

Let $A, A^{\prime}$ be symmetric $\mathcal{O}$-algebras. Following Broué [2, §5] an $A$ - $A^{\prime}$-bimodule $M$ is said to induce a stable equivalence of Morita type if $M$ is finitely generated projective as a left $A$-module, as a right $A^{\prime}$-module, and if $M \otimes_{A^{\prime}} M^{*} \cong A \oplus U$ for some projective $A$ - $A$-bimodule and $M^{*} \otimes_{A} M \cong A^{\prime} \oplus U^{\prime}$ for some projective $A^{\prime}$ - $A^{\prime}$-bimodule $U^{\prime}$. In that case, the functors $M \otimes_{A^{\prime}}-$ and $M^{*} \otimes_{A}-$ induce inverse equivalences $\underline{\bmod }(A) \cong \bmod \left(A^{\prime}\right)$. Stable equivalences of Morita type can be composed by tensoring the relevant bimodules together. In particular, a stable equivalence of Morita type between two block algebras induces a stable equivalence of Morita type between any two source algebras of these blocks.

A stable equivalence of Morita type between two block algebras $\mathcal{O} G b, \mathcal{O} G^{\prime} b^{\prime}$ is called splendid if it is induced by a $p$-permutation bimodule. In that case the defect groups of $b$ and $b^{\prime}$ are isomorphic, and for some identification of a defect group $P$ of $b$ with a defect group of $b^{\prime}$ there is a choice of source algebras $A$ and $A^{\prime}$ such that the induced stable equivalence of Morita type between $A$ and $A^{\prime}$ is given by an $A$ - $A^{\prime}$-bimodule which is an indecomposable direct summand of $A \otimes_{\mathcal{O} P} A^{\prime}$. By [9, 7.6.6] the fusion systems of $A$ and $A^{\prime}$ are then equal (see also [4, 3.1] for a proof of this fact).

Theorem 1.5. Let $G, G^{\prime}$ be finite groups, $b$ a block of $\mathcal{O} G$, and $b^{\prime}$ a block of $\mathcal{O} G^{\prime}$. $A$ splendid stable equivalence of Morita type between $\mathcal{O} G b$ and $\mathcal{O} G b^{\prime}$ induces an 
equivalence of categories

$$
\operatorname{coMack}_{0}(G, b) \cong \operatorname{coMack}_{0}\left(G^{\prime}, b^{\prime}\right) .
$$

Let $\mathcal{A}$ be an abelian category with enough projective objects. The projective dimension $\operatorname{pdim}(U)$ of an object $U$ in $\mathcal{A}$ is the smallest nonnegative integer $n$ for which there exists a projective resolution which is zero in all degrees larger than $n$, provided that $U$ has a bounded projective resolution, and $\operatorname{pdim}(U)=\infty$ otherwise. The finitistic dimension of $\mathcal{A}$ is the supremum of the set of integers $\operatorname{pdim}(U)$, with $U$ running over all objects in $\mathcal{A}$ having a finite projective dimension (and with the convention that the finitistic dimension is $\infty$ if this supremum is unbounded). The left or right finitistic dimension of a finite-dimensional $k$-algebra is the finitistic dimension of its category of finitely generated left or right modules, respectively. The $p$-sectional rank $s(G)$ of a finite group $G$ is the maximum of the ranks of $p$-elementary abelian subquotients of $G$. Tambara showed in 11] that $1+s(G)$ is equal to the finitistic dimension of $\operatorname{coMack}(k G)$. We show here, essentially by adapting Tambara's proof, that there is an analogous statement for block algebras.

Theorem 1.6. Let $G$ be a finite group, $b$ a block of $k G$ and $P$ a defect group of $b$. The finitistic dimension of $\operatorname{coMack}(G, b)$ is equal to $1+s(P)$.

Remark 1.7. By results of Thévenaz and Webb in [12, §17], the category $\operatorname{Mack}(k G)$ of all Mackey functors on $G$ over $k$ has a block decomposition which corresponds bijectively to the block decomposition of $k G$. It seems however unknown whether the blocks of $\operatorname{Mack}(k G)$ admit a description in terms of the source algebras of the corresponding blocks of $k G$. In contrast to Theorem 1.6. Bouc, Stancu and Webb [1. Theorem 1.2] showed that the finitistic dimension of $\operatorname{Mack}(k G)$ is zero.

The proofs of the above theorems will be given in the last section. If not stated otherwise, modules are unital left modules.

\section{Derived EQuivalences AND ENDOMORPhism ALGEBRAS}

An $\mathcal{O}$-algebra $A$ is called symmetric if $A$ is isomorphic to its $\mathcal{O}$-dual $A^{*}$ as an $A$ - $A$-bimodule. If $s \in A^{*}$ is the image of $1_{A}$ under some bimodule isomorphism $A \cong A^{*}$, then $s$ is called a symmetrising form on $A$. Matrix algebras, finite group algebras over $\mathcal{O}$, their block algebras and source algebras are symmetric.

For $A$ an $\mathcal{O}$-algebra, $(U, \delta)$ a complex of right $A$-modules and $(V, \epsilon)$ a complex of left $A$-modules we denote by $U \otimes_{A} V$ the complex of $\mathcal{O}$-modules which in degree $n$ is equal to $\bigoplus_{(i, j)} U_{i} \otimes_{A} V_{j}$, with $(i, j)$ running over the set of pairs of integers satisfying $i+j=n$. The differential of $U \otimes_{A} V$ is the sum of the maps $\operatorname{Id}_{U_{i}} \otimes \epsilon_{j}$ and $(-1)^{i} \delta_{i} \otimes \operatorname{Id}_{V_{j}}$ from $U_{i} \otimes_{A} V_{j}$ to $U_{i} \otimes_{A} V_{j-1}$ and to $U_{i-1} \otimes_{A} V_{j}$. If $U, V$ are bimodule complexes, then $U \otimes_{A} V$ inherits those bimodule structures.

For $(V, \delta)$ and $(W, \zeta)$ two complexes of $A$-modules, we denote by $\operatorname{Hom}_{A}(V, W)$ the complex of $\mathcal{O}$-modules which in degree $n$ is equal to $\bigoplus_{(i, j)} \operatorname{Hom}_{A}\left(V_{i}, W_{j}\right)$, with $(i, j)$ running over the pairs of integers satisfying $j-i=n$. The differential is the sum of the maps from $\operatorname{Hom}_{A}\left(V_{i}, W_{j}\right)$ to $\operatorname{Hom}_{A}\left(V_{i}, W_{j-1}\right)$ and to $\operatorname{Hom}_{A}\left(V_{i+1}, W_{j}\right)$, where the first is given by composition with $\zeta_{j}$, and the second by precomposition with $(-1)^{n+1} \epsilon_{i+1}$. As before, if $V, W$ have bimodule structures, then these induce bimodule structures on the complex $\operatorname{Hom}_{A}(V, W)$. Note that this sign convention implies that the $k$-dual $V^{*}$ of the complex $V$ is the complex which in degree $n$ is equal to $V_{-n}^{*}$, with differential $V_{-n}^{*} \rightarrow V_{-n+1}^{*}$ given by precomposing 
with $(-1)^{n+1} \epsilon_{-n+1}: V_{-n+1} \rightarrow V_{-n}$. If $A$ is symmetric and $s \in A^{*}$ is a symmetrising form of $A$, then composition with $s$ induces an isomorphism of complexes $\operatorname{Hom}_{A}(V, A) \cong V^{*}$.

For $A, A^{\prime}$ symmetric $k$-algebras, a Rickard complex of $A$ - $A^{\prime}$-bimodules is a bounded complex $X$ of $A$ - $A^{\prime}$-bimodules whose terms are finitely generated projective as left $A$-modules and as right $A^{\prime}$-modules, such that we have homotopy equivalences $X \otimes_{A^{\prime}} X^{*} \simeq A$ and $X^{*} \otimes_{A} X \simeq A^{\prime}$ as complexes of $A$ - $A$-bimodules and $A^{\prime}$ - $A^{\prime}$-bimodules, respectively.

Theorem 2.1. Let $A, A^{\prime}$ be symmetric $k$-algebras. Let $N$ be a finitely generated $A$-module, and let $N^{\prime}$ be a finitely generated $A^{\prime}$-module. Set $E=\operatorname{End}_{A}(N)$ and $E^{\prime}=\operatorname{End}_{A^{\prime}}\left(N^{\prime}\right)$. Let $X$ be a Rickard complex of $A$ - $A^{\prime}$-bimodules. Suppose that every indecomposable direct summand of every term of $X \otimes_{A^{\prime}} N^{\prime}$ is isomorphic to a direct summand of $N$ and that every indecomposable direct summand of every term of $X^{*} \otimes_{A} N$ is isomorphic to a direct summand of $N^{\prime}$. Set $Y=\operatorname{Hom}_{A}\left(X \otimes_{A^{\prime}} N^{\prime}, N\right)$ and $Y^{\prime}=\operatorname{Hom}_{A^{\prime}}\left(X^{*} \otimes_{A} N, N^{\prime}\right)$. Then $Y$ is a complex of E-E'-bimodules, $Y^{\prime}$ is a complex of $E^{\prime}$-E-bimodules, the terms of $Y$ and $Y^{\prime}$ are finitely generated projective as left and as right modules, and we have homotopy equivalences

$$
\begin{aligned}
& Y \otimes_{E^{\prime}} Y^{\prime} \simeq E, \\
& Y^{\prime} \otimes_{E} Y \simeq E^{\prime}
\end{aligned}
$$

as complexes of E-E-bimodules and $E^{\prime}-E^{\prime}$-bimodules, respectively. In particular, $E$ and $E^{\prime}$ are derived equivalent. Moreover, if $X$ and $X^{*}$ induce a Morita equivalence between $A$ and $A^{\prime}$, then $Y$ and $Y^{\prime}$ induce a Morita equivalence between $E$ and $E^{\prime}$.

Proof. If $i, j$ are idempotents in $A$, then we have a canonical isomorphism $i A \otimes_{A}$ $A j \cong i A j$, induced by multiplication in $A$. We translate this to endomorphism algebras. Let $U, V$ be direct summands of $N$. Let $\tau$ be a projection of $N$ onto $U$, viewed as an idempotent in $E$, and let $\sigma$ be a projection of $N$ onto $V$. Then $\operatorname{Hom}_{A}(U, N) \cong$ $E \circ \tau$ is a projective left $E$-module via composition of homomorphisms. Similarly, $\operatorname{Hom}_{A}(N, V) \cong \sigma \circ E$ is a projective right $E$-module via precomposition by homomorphisms in $E$. Thus the first observation applied to $E$ instead of $A$ implies that composition of homomorphisms induces an isomorphism

$$
\operatorname{Hom}_{A}(N, V) \otimes_{E} \operatorname{Hom}_{A}(U, N) \cong \operatorname{Hom}_{A}(U, V)
$$

which is natural and additive in $U$ and $V$. Thus this isomorphism holds for $U$ and $V$ finite direct sums of direct summands of $N$. Similar statements hold for $A^{\prime}$ and $N^{\prime}$. By the assumptions, the indecomposable direct summands of the terms of $X^{*} \otimes_{A} N$ are isomorphic to direct summands of $N^{\prime}$. Thus the above isomorphism, applied with $U=V=X^{*} \otimes_{A} N$ and $N^{\prime}$ instead of $N$, shows that composition of homomorphisms yields an isomorphism

$$
\operatorname{Hom}_{A^{\prime}}\left(N^{\prime}, X^{*} \otimes_{A} N\right) \otimes_{E^{\prime}} \operatorname{Hom}_{A^{\prime}}\left(X^{*} \otimes_{A} N, N^{\prime}\right) \cong \operatorname{End}_{A^{\prime}}\left(X^{*} \otimes_{A} N\right)
$$

as graded $E$-E-bimodules. A straightforward verification (checking signs) shows that this isomorphism commutes with the differentials, so this is an isomorphism of complexes of $E$-E-bimodules. By a standard adjunction, the complex on the left side of the tensor product is isomorphic to $\operatorname{Hom}_{A}\left(X \otimes_{A^{\prime}} N^{\prime}, N\right)=Y$. By another standard adjunction, the right side of the above isomorphism is isomorphic to the complex

$$
\operatorname{Hom}_{A}\left(N, X \otimes_{A^{\prime}} X^{*} \otimes_{A} N\right) .
$$


Since $X$ is a Rickard complex, we have $X \otimes_{A^{\prime}} X^{*} \otimes_{A} N \simeq N$, and hence

$$
\operatorname{Hom}_{A}\left(N, X \otimes_{A^{\prime}} X^{*} \otimes_{A} N\right) \simeq \operatorname{Hom}_{A}(N, N)=E .
$$

This shows that $Y \otimes_{E^{\prime}} Y^{\prime} \simeq E$. A similar argument shows that $Y^{\prime} \otimes_{E} Y \simeq E^{\prime}$, which completes the proof.

\section{ON THE FINITISTIC DIMENSION OF ENDOMORPHISM ALGEBRAS}

The result of this section holds regardless of the characteristic of the field $k$. Let $A$ be a finite-dimensional $k$-algebra and $U$ a finitely generated $A$-module. We denote by $\operatorname{add}(U)$ the full additive subcategory of all $A$-modules which are isomorphic to finite direct sums of direct summands of $U$. We use, without further reference, the following standard facts. If $V$ belongs to add $(U)$, then $\operatorname{Hom}_{A}(U, V)$ is a projective right $\operatorname{End}_{A}(U)$-module, and any finitely generated projective right End $_{A}(U)$-module is of this form, up to isomorphism. Given two idempotents $i, j$ in $A$, every homomorphism of right $A$-modules $i A \rightarrow j A$ is induced by left multiplication with an element in $j A i$. Multiplication by $i$ is exact; in particular, if $Z$ is a complex of $A$-modules which is exact or which has homology concentrated in a single degree, the same is true for finite direct sums of complexes of the form $i Z$. Translated to endomorphism algebras this implies that for any two $A$-modules $V, W$ in $\operatorname{add}(U)$, any homomorphism of right $\operatorname{End}_{A}(U)$-modules $\operatorname{Hom}_{A}(U, V) \rightarrow$ $\operatorname{Hom}_{A}(U, W)$ is induced by composition with an $A$-homomorphism $V \rightarrow W$. Thus any complex of finitely generated projective right $\operatorname{End}_{A}(U)$-modules is isomorphic to a complex obtained from applying the functor $\operatorname{Hom}_{A}(U,-)$ to a complex of $A$ modules $Z$ whose terms belong to $\operatorname{add}(U)$. Moreover, if $\operatorname{Hom}_{A}(U, Z)$ is exact or has homology concentrated in a single degree, the same is true for any complex of the form $\operatorname{Hom}_{A}\left(U^{\prime}, Z\right)$, where $U^{\prime} \in \operatorname{add}(U)$.

Let $A$ and $B$ be symmetric $k$-algebras. Following [6], an $A$-B-bimodule $M$ is said to induce a separable equivalence between $A$ and $B$ if $M$ is finitely generated projective as a left $A$-module, as a right $B$-module, and if $A$ is isomorphic to a direct summand of $M \otimes_{B} M^{*}$ as an $A$-A-bimodule and $B$ is isomorphic to a direct summand of $M^{*} \otimes_{A} M$ as a $B$ - $B$-bimodule. If there is such a bimodule, we say that $A$ and $B$ are separably equivalent. The link between this section and block theory comes from the fact that a block algebra of a finite group is separably equivalent to its defect group algebras via a $p$-permutation bimodule. Since the notion of separable equivalence is transitive, any two block algebras with isomorphic defect groups are separably equivalent via $p$-permutation bimodules.

Theorem 3.1. Let $A, B$ be symmetric $k$-algebras. Let $M$ be an $A$-B-bimodule inducing a separable equivalence between $A$ and $B$. Let $U$ be a finitely generated $A$-module and $V$ a finitely generated $B$-module. Suppose that $M^{*} \otimes_{A} U \in \operatorname{add}(V)$ and that $M \otimes_{B} V \in \operatorname{add}(U)$. Then the right finitistic dimensions of $\operatorname{End}_{A}(U)$ and of $\operatorname{End}_{B}(V)$ are equal.

Proof. Set $E=\operatorname{End}_{A}(U)$ and $F=\operatorname{End}_{B}(V)$. We show first that $\operatorname{add}\left(M \otimes_{B} V\right)=$ $\operatorname{add}(U)$ and $\operatorname{add}\left(M^{*} \otimes_{A} U\right)=\operatorname{add}(V)$. By the assumptions, we have $\operatorname{add}\left(M \otimes_{B} V\right) \subseteq$ $\operatorname{add}(U)$. Thus $\operatorname{add}\left(M^{*} \otimes_{A} M \otimes_{B} V\right) \subseteq \operatorname{add}\left(M^{*} \otimes_{A} U\right) \subseteq \operatorname{add}(V)$. Since $B$ is isomorphic to a direct summand of the $B$-B-bimodule $M^{*} \otimes_{A} M$, it follows that $V$ is isomorphic to a direct summand of $M^{*} \otimes_{A} M \otimes_{B} V$. Thus the previous inclusions of additive categories are equalities. The same argument with reversed roles shows the second equality. We show next that for any finitely generated right $F$-module 
of finite projective dimension there is a finitely generated right $E$-module with the same projective dimension. Let $Y$ be a right $F$-module with a finite projective $\operatorname{dimension} \operatorname{pdim}(Y)=n$. By the remarks at the beginning of this section, any such resolution is of the form

$$
0 \rightarrow \operatorname{Hom}_{B}\left(V, V_{n}\right) \rightarrow \cdots \rightarrow \operatorname{Hom}_{B}\left(V, V_{1}\right) \rightarrow \operatorname{Hom}_{B}\left(V, V_{0}\right) \rightarrow Y \rightarrow 0
$$

obtained from applying the functor $\operatorname{Hom}_{B}(V,-)$ to a complex of $B$-modules

$$
Z=\cdots \rightarrow 0 \rightarrow V_{n} \rightarrow \cdots \rightarrow V_{1} \rightarrow V_{0} \rightarrow 0 \rightarrow \cdots
$$

whose components $V_{i}$ are in $\operatorname{add}(V)$, together with a surjective homomorphism of right $F$-modules $\operatorname{Hom}_{B}\left(V, V_{0}\right) \rightarrow Y$. Thus the complex $\operatorname{Hom}_{B}(V, Z)$ has homology concentrated in degree 0 , and the homology in degree 0 is $Y$. By the remarks at the beginning of this section, it follows that for any $B$-module $V^{\prime}$ in $\operatorname{add}(V)$, applying the functor $\operatorname{Hom}_{B}\left(V^{\prime},-\right)$ to the complex of $B$-modules $Z$ yields a complex of $k$-vector spaces

$$
\operatorname{Hom}_{B}\left(V^{\prime}, Z\right)
$$

which has nonzero homology only in degree 0 , if any. By the assumptions, this applies in particular to the $B$-module $V^{\prime}=M^{*} \otimes_{A} M \otimes_{B} V$. Using a standard adjunction, we obtain a complex of the form

$$
\operatorname{Hom}_{A}\left(M \otimes_{B} V, M \otimes_{B} Z\right)
$$

which has homology concentrated in degree 0 . This is a complex of right $\operatorname{End}_{A}\left(M \otimes_{B} V\right)$-modules. Since $\operatorname{add}\left(M \otimes_{B} V\right)=\operatorname{add}(U)$, it follows that the complex

$$
\operatorname{Hom}_{A}\left(U, M \otimes_{B} Z\right)
$$

has homology concentrated in degree 0 . This is a complex of right $E$-modules. Its homology in degree 0 is therefore a right $E$-module $X$ with projective dimension at most $n$. We show that $X$ has projective dimension exactly $n$. If $n=0$, there is nothing to prove, so assume that $n>0$. The fact that $\operatorname{pdim}(Y)=n$ implies in particular that the first nonzero differential $\operatorname{Hom}_{B}\left(V, V_{n}\right) \rightarrow \operatorname{Hom}_{B}\left(V, V_{n-1}\right)$ in the complex $\operatorname{Hom}_{B}(V, Z)$ is not split injective. Thus the first nonzero differential $V_{n} \rightarrow$ $V_{n-1}$ in $Z$ is not split injective. But then the first nonzero differential of $M \otimes_{B} Z$ is not split injective either, for if it were, so would be that of $M^{*} \otimes_{A} M \otimes_{B} Z$, which is not possible, as $Z$ is a direct summand, as a complex of $B$-modules, of $M^{*} \otimes_{A} M \otimes_{B} Z$. This in turn implies that the first nonzero differential of $\operatorname{Hom}_{A}\left(U, M \otimes_{B} Z\right)$ is not split injective as a right $E$-module homomorphism. This shows that $\operatorname{pdim}(X)=\operatorname{pdim}(Y)$. Thus the right finitistic dimension of $E$ is at least as large as that of $F$. Exchanging the roles of $E$ and $F$ shows the equality of right finitistic dimensions as stated.

\section{Proofs}

Let $A$ be an $\mathcal{O}$-algebra which is finitely generated as an $\mathcal{O}$-module. Let $U, U^{\prime}$ be finitely generated $A$-modules. An idempotent in $\operatorname{End}_{A}(U)$ corresponds to a projection of $U$ onto a direct summand of $U$. The Krull-Schmidt theorem implies that this correspondence induces a bijection between conjugacy classes of primitive idempotents in $\operatorname{End}_{A}(U)$ and isomorphism classes of indecomposable direct summands of $U$. Thus if $U$ and $U^{\prime}$ have the same isomorphism classes of indecomposable direct summands, or equivalently, if $\operatorname{add}(U)=\operatorname{add}\left(U^{\prime}\right)$, then $\operatorname{End}_{A}(U)$ and $\operatorname{End}_{A}\left(U^{\prime}\right)$ are Morita equivalent. We will use this well-known fact without further reference. 
Proof of Theorem 1.1. Let $G$ be a finite group, $b$ a block of $\mathcal{O} G, P$ a defect group of $b$, and $i \in(\mathcal{O} G b)^{P}$ a source idempotent of $b$. By [7, 3.5], multiplication with $i$ induces a Morita equivalence between $\mathcal{O} G b$ and the source algebra $A=i \mathcal{O} G i$. By Yoshida's theorem [13, 4.3] mentioned above, the category $\operatorname{coMack}(G, b)$ is equivalent to $\bmod \left(\operatorname{End}_{\mathcal{O} G b}\left(\oplus_{H} b \cdot \mathcal{O} G / H\right)^{\mathrm{op}}\right)$, where $H$ runs over the subgroups of $G$. Fix a subgroup $H$ of $G$, and let $U$ be an indecomposable direct summand of $b \cdot \mathcal{O} G / H \cong$ $b \cdot \operatorname{Ind}_{H}^{G}(\mathcal{O})$. Then $U$ has trivial source. By [3, 6.1] there exists a vertex $Q$ of $U$ such that $U$ is isomorphic to a direct summand of $\mathcal{O} G i \otimes_{\mathcal{O} Q} \mathcal{O} \cong \mathcal{O} G i \otimes_{A} A \otimes_{\mathcal{O} Q} \mathcal{O}$. Thus $U \cong \mathcal{O} G i \otimes_{A} V$ for some indecomposable direct summand $V$ of $A \otimes_{\mathcal{O} Q} \mathcal{O}$. Conversely, if $W$ is an indecomposable direct summand of $N$, then $\mathcal{O} G i \otimes_{A} W$ is isomorphic to a direct summand of $\mathcal{O} G / Q$ for some subgroup $Q$ of $P$. This implies that $\operatorname{End}_{\mathcal{O} G b}\left(\oplus_{H} b \cdot \mathcal{O} G / H\right)^{\mathrm{op}}$ is Morita equivalent to $\operatorname{End}_{\mathcal{O} G b}\left(\mathcal{O} G i \otimes_{A} N\right)^{\mathrm{op}}$, where $H$ runs over the subgroups of $G$. Since multiplication by $i$ induces a Morita equivalence between $\mathcal{O} G b$ and $A$ it follows that the last algebra is isomorphic to $\operatorname{End}_{A}(N)^{\mathrm{op}}=E^{\mathrm{op}}$, whence the equivalence $\operatorname{coMack}(G, b) \cong \bmod \left(E^{\mathrm{op}}\right)$. Let $A^{\prime}$ be a source algebra of a block $b^{\prime}$ of a finite group $G^{\prime}$ having $P$ as a defect group. Set $N^{\prime}=\bigoplus_{Q} A^{\prime} \otimes_{\mathcal{O} Q} \mathcal{O}$, where $Q$ runs over the subgroups of $P$. By the above, a Morita equivalence between $A$ and $A^{\prime}$ which induces a bijection between the isomorphism classes of indecomposable direct summand of $N$ and of $N^{\prime}$ induces an equivalence $\operatorname{coMack}(G, b) \cong \operatorname{coMack}\left(G^{\prime}, b^{\prime}\right)$.

Proof of Theorem 1.2. We show that this is a special case of 2.1. Let $Q$ be a subgroup of $P$. We need to show that every indecomposable direct summand of one of the terms of the complex of $A$-modules $X \otimes_{A^{\prime}} A \otimes_{\mathcal{O} Q} \mathcal{O} \cong X \otimes_{\mathcal{O} Q} \mathcal{O}$ is isomorphic to a direct summand of $A \otimes_{\mathcal{O} R} \mathcal{O}$ for some subgroup $R$ of $P$. Since the terms of $X$ are finite direct sums of summands of bimodules of the form $A \otimes_{\mathcal{O}} A^{\prime}$, with $S$ running over the subgroups of $P$, it suffices to show that for $S$ a subgroup of $P$, any indecomposable direct summand $U$ of the left $A$-module $A \otimes_{\mathcal{O} S} A^{\prime} \otimes_{k Q} \mathcal{O}$ is isomorphic to a direct summand of $A \otimes_{\mathcal{O} R} \mathcal{O}$ for some subgroup $R$. Since $A^{\prime}$ is a permutation $\mathcal{O} P-\mathcal{O} P$-module which is finitely generated projective as a left and right $\mathcal{O} P$-module, it follows that any indecomposable direct summand of $A^{\prime}$ as an $\mathcal{O} Q-\mathcal{O} S$-bimodule is isomorphic to $\mathcal{O} S \otimes_{\mathcal{O} R}\left({ }_{\psi} \mathcal{O} Q\right)$ for some injective group homomorphism $\psi: R \rightarrow Q$. (See [4, Appendix] for a review on the use of the bimodule structure of source algebras, going back to work of Puig [8].) Thus $U$ is isomorphic to a direct summand of a left $A$-module of the form $A \otimes_{\mathcal{O} S} \mathcal{O} S \otimes_{\mathcal{O} R}$ $\left({ }_{\psi} \mathcal{O} Q\right) \otimes_{\mathcal{O} Q} \mathcal{O} \cong A \otimes_{\mathcal{O} R} \mathcal{O}$ as claimed. Exchanging the roles of $A$ and $A^{\prime}$ completes the proof of the first statement. Let the last statement follow from this, together with the description in 1.1 of cohomological Mackey functors in terms of source algebras.

Proof of Theorem 1.3. Let $G$ be a finite group, $b$ a block of $\mathcal{O} G$, and $P$ a defect group of $b$. Let $N$ and $\underline{E}$ be as in the statement. Since $N$ is $\mathcal{O}$-free, it follows that an $A$-endomorphism $\varphi$ of $N$ factors through a relatively $\mathcal{O}$-projective $A$-module if and only if it factors through a projective $A$-module. This is in turn equivalent to stating that $\varphi$ is a sum of endomorphisms which factor through the summand $A \otimes_{\mathcal{O}} \mathcal{O}$ of $N$. It follows that $\operatorname{End}_{A}^{\mathrm{pr}}(N)$ is equal to the ideal in $E=\operatorname{End}_{A}(N)$ generated by the projection $\tau$ of $N$ onto $A \otimes_{\mathcal{O}} \mathcal{O}$. Thus an $E$-module $U$ is the

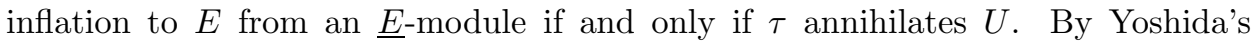


translation of $E^{\mathrm{op}}$-modules into cohomological Mackey functors in [13, the $E^{\mathrm{op}}$ modules annihilated by $\tau$ correspond exactly to the cohomological Mackey functors which vanish at the trivial subgroup. This shows the equivalence of categories $\operatorname{coMack}_{0}(G, b) \cong \bmod \left(\underline{E}^{\mathrm{op}}\right)$.

Proof of Corollary 1.4. With the notation of the previous proof, we have

$$
\underline{E}=E / E \circ \tau \circ E \text {. }
$$

We also have $\tau \circ E \circ \tau=\operatorname{End}_{A}(A) \cong A^{\mathrm{op}}$. Thus we have a standard recollement determined by the idempotent $\tau$ in $E$. The result follows, using the Morita equivalences between $A, \bmod \left(E^{\mathrm{op}}\right), \bmod \left(\underline{E}^{\mathrm{op}}\right)$ and $\mathcal{O} G b, \operatorname{coMack}(G, b)$, and $\operatorname{coMack}_{0}(G, b)$ from [7, 3.5], 1.1, 1.3, respectively.

We will need the following variation of a fact stated at the beginning of this section: the idempotents in the kernel of the canonical algebra homomorphism $\operatorname{End}_{A}(U) \rightarrow \underline{\text { End }}_{A}(U)$ correspond exactly to the relatively $\mathcal{O}$-projective indecomposable direct summands of $U$. Thus if two finitely generated $A$-modules $U$ and $U^{\prime}$ have the same isomorphism classes of indecomposable direct summands which are not relatively $\mathcal{O}$-projective, then $\operatorname{End}_{A}(U)$ and $\underline{\operatorname{End}}_{A}\left(U^{\prime}\right)$ are Morita equivalent.

Proof of Theorem 1.5. Suppose that there is a splendid stable equivalence of Morita type between $\mathcal{O} G b$ and $\mathcal{O} G^{\prime} b^{\prime}$. As mentioned in the introduction, it follows from [9, 7.6.6] or [4, 3.1] that there is an identification of $P$ with a defect group of $b^{\prime}$ and that there are source algebras $A$ of $\mathcal{O} G b$ and $A^{\prime}$ of $\mathcal{O} G^{\prime} b^{\prime}$ such that the induced stable equivalence of Morita type between $A$ and $A^{\prime}$ is given by a bimodule $M$ which is an indecomposable direct summand of $A \otimes_{\mathcal{O} P} A^{\prime}$. Set $N=\bigoplus_{Q} A \otimes_{\mathcal{O} Q} \mathcal{O}$ and $N^{\prime}=$ $\bigoplus_{Q} A^{\prime} \otimes_{\mathcal{O} Q} \mathcal{O}$, where in the direct sums $Q$ runs over the subgroups of $P$. Set $E=$ $\operatorname{End}_{A}(N)$ and $\underline{E}=\underline{\operatorname{End}}_{A}(N)$. Similarly, set $E^{\prime}=\operatorname{End}_{A^{\prime}}\left(N^{\prime}\right)$ and $\underline{E}^{\prime}=\underline{\operatorname{End}}_{A^{\prime}}\left(N^{\prime}\right)$. By 1.3, in order to show that $\operatorname{coMack}_{0}(G, b) \cong \operatorname{coMack}_{0}\left(G^{\prime}, b^{\prime}\right)$ it suffices to show that $\bmod (\underline{E}) \cong \bmod \left(\underline{E}^{\prime}\right)$. Since $M$ induces a stable equivalence of Morita type, it follows that we have an algebra isomorphism $\underline{\operatorname{End}}_{A}\left(M \otimes_{A^{\prime}} N^{\prime}\right) \cong \underline{E}^{\prime}$. Thus it suffices to show that the $A$-modules $N$ and $M \otimes_{A^{\prime}} N^{\prime}$ have the same isomorphism classes of indecomposable nonprojective direct summands.

Let $V^{\prime}$ be an indecomposable nonprojective direct summand of $N^{\prime}$. Then there is a subgroup $Q$ of $P$ such that $V^{\prime}$ is an indecomposable nonprojective direct summand of $A^{\prime} \otimes_{\mathcal{O} Q} V^{\prime}$. It follows that $M \otimes_{A^{\prime}} V^{\prime}$ has, up to isomorphism, a unique indecomposable nonprojective direct summand $V$, which is by construction isomorphic to a direct summand of $A \otimes_{\mathcal{O} P} A^{\prime} \otimes_{\mathcal{O} Q} \mathcal{O}$. The argument used in the proof of Theorem 1.2 above shows that $V$ is a summand of $A \otimes_{\mathcal{O} R} \mathcal{O}$ for some subgroup $R$ of $P$, hence $V$ is isomorphic to a summand of $N$. Exchanging the roles of $N$ and $N^{\prime}$ as well as $M$ and $M^{*}$ implies that the functors $M \otimes_{A^{\prime}}-$ and $M^{*} \otimes_{A}$ - induce a bijection between the isomorphism classes of indecomposable nonprojective direct summands of $N$ and $N^{\prime}$. The result follows.

Proof of Theorem 1.6. Denote by $A$ a source algebra of $k G b$ for the defect group $P$. It is well known that $A$ and the defect group algebra $k P$ are separably equivalent via the $A$ - $k P$-bimodule $A_{P}$, obtained from restricting the $A$ - $A$-bimodule $A$ on the right side to $k P$ via the canonical injection $k P \rightarrow A$ (see e.g. [5, 4.2]). Since $A$ is symmetric, the dual of $A_{P}$ is isomorphic to ${ }_{P} A$, obtained from restricting the $A-A$ bimodule $A$ to $k P$ on the left side. By Tambara's main result in [11, the finitistic 
dimension of $\operatorname{coMack}(k P)$ is equal to $1+s(P)$. Set $V=\bigoplus_{Q \leq P} k P / Q$ and $U=$ $A \otimes_{k P} V=\bigoplus_{Q \leq P} A \otimes_{k Q} k$. We have $\left({ }_{P} A\right) \otimes_{A} U=\operatorname{Res}_{P}(U)$. This is a permutation $k P$-module, because $A$ has a $P \times P$-stable $k$-basis. Thus the functor $A \otimes_{k P}-$ sends $V$ to add $(U)$, and the functor ${ }_{P} A \otimes_{A}-$ sends $U$ to $\operatorname{add}(V)$. It follows from Theorem 3.1 that $\operatorname{End}_{k P}(V)$ and $\operatorname{End}_{A}(U)$ have the same right finitistic dimension. Since the categories of finitely generated right modules over $\operatorname{End}_{k P}(V)$ and $\operatorname{End}_{A}(U)$ are equivalent to $\operatorname{coMack}(k P)$ and $\operatorname{coMack}(G, b)$, respectively, by Yoshida's theorem [13. 4.3] and 1.1 above, it follows that the finitistic $\operatorname{dimension}$ of $\operatorname{coMack}(G, b)$ is $1+s(P)$ as stated.

\section{REFERENCES}

[1] S. Bouc, R. Stancu, and P. J. Webb, On the projective dimensions of Mackey functors, preprint (2015), arXiv:1503.03955

[2] M. Broué, Equivalences of blocks of group algebras, Finite-dimensional algebras and related topics (Ottawa, ON, 1992), NATO Adv. Sci. Inst. Ser. C Math. Phys. Sci., vol. 424, Kluwer Acad. Publ., Dordrecht, 1994, pp. 1-26, DOI 10.1007/978-94-017-1556-0_1. MR.1308978

[3] M. Linckelmann, The source algebras of blocks with a Klein four defect group, J. Algebra 167 (1994), no. 3, 821-854, DOI 10.1006/jabr.1994.1214. MR.1287072

[4] M. Linckelmann, On splendid derived and stable equivalences between blocks of finite groups, J. Algebra 242 (2001), no. 2, 819-843, DOI 10.1006/jabr.2001.8812. MR.1848975

[5] M. Linckelmann, Trivial source bimodule rings for blocks and p-permutation equivalences, Trans. Amer. Math. Soc. 361 (2009), no. 3, 1279-1316, DOI 10.1090/S0002-9947-08-04577-7. MR2457399

[6] M. Linckelmann, Finite generation of Hochschild cohomology of Hecke algebras of finite classical type in characteristic zero, Bull. Lond. Math. Soc. 43 (2011), no. 5, 871-885, DOI 10.1112/blms/bdr024. MR2854558

[7] L. Puig, Pointed groups and construction of characters, Math. Z. 176 (1981), no. 2, 265-292, DOI 10.1007/BF01261873. MR607966

[8] L. Puig, Local fusions in block source algebras, J. Algebra 104 (1986), no. 2, 358-369, DOI 10.1016/0021-8693(86)90221-8. MR866781

[9] L. Puig, On the local structure of Morita and Rickard equivalences between Brauer blocks, Progress in Mathematics, vol. 178, Birkhäuser Verlag, Basel, 1999. MR.1707300

[10] B. Rognerud, Equivalences between blocks of cohomological Mackey algebras, Math. Z. 280 (2015), no. 1-2, 421-449, DOI 10.1007/s00209-015-1431-x. MR3343914

[11] D. Tambara, Homological properties of the endomorphism rings of certain permutation modules, Osaka J. Math. 26 (1989), no. 4, 807-828. MR.1040426

[12] J. Thévenaz and P. Webb, The structure of Mackey functors, Trans. Amer. Math. Soc. 347 (1995), no. 6, 1865-1961, DOI 10.2307/2154915. MR.1261590

[13] T. Yoshida, On G-functors. II. Hecke operators and G-functors, J. Math. Soc. Japan 35 (1983), no. 1, 179-190, DOI 10.2969/jmsj/03510179. MR679083

City University London, Department of Mathematics, London EC1V OHB

E-mail address: Markus.Linckelmann.1@city.ac.uk 